\title{
Analysis of balance control methods based on inverted pendulum for legged robots
}

\author{
Alexander Denisov*1, Roman Iakovlev ${ }^{1}$, Ilhshat Mamaev² and Nikita Pavliuk ${ }^{1}$ \\ ${ }^{1}$ SPIIRAS, Laboratory of Autonomous Robotic Systems, 199178 Saint-Petersburg, 14-th line of V.I., 39, Russia \\ ${ }^{2}$ KIT, Institute for Anthropomatics and Robotics, Intelligent Process Automation and Robotics Lab, Engler-Bunte-Ring 8, D-76131, \\ Karlsruhe, Germany
}

\begin{abstract}
Methods of balance control for a legged robot, the model of which is presented as a two-section inverted pendulum, are considered. The following balance methods for humanoid robots are analysed: the parallel algorithm of the network operator method; the method of natural synergies; the method of fuzzy control, the spherical inverted pendulum mode, a dual length linear inverted pendulum method. The best of these methods will be used in the development of the Russian anthropomorphic robot Antares.
\end{abstract}

\section{Introduction}

The development of walking robots is a process consisting of many tasks, among which one of the most important is to ensure the stability of the position and movement of walking robots. This task is especially important in the development of anthropomorphic robots that have only two points of support when moving in space.

The balance problem is actual for legged robot. A lot of different methods of solving this problem exist for robots with different number of legs. There are some of them:

In the paper [1] general Segway model under control based on Riccati equation for solving stabilization problems of four-legged robot RQuad during of walking, running, jumping is used.

In the paper [2] the transitional process of two-legged robot, which begins when the style of move is changed from walking to running and conversely is considered. Proposed approach based on anthropomorphic kinematics provides smooth move speed increase and robust movement when transition from walking to running appears.

In the paper [3] the walking control method for hexapod robot is considered. This method provides robust movements to different sides.

In the paper [4] modeling of movement for fourlegged robot and visualization of its walking parameters, which provide to define main balance problems for legged robots, are executed in MatLab, SimMechanics.

In the paper [5] the planning of humanoid robot walking based on solving of contact planning problem is described. The results of testing this method on humanoid robot HRP-2 is a multi-contact motion by relaxing the contacts to occur with any robot and environment.
In the paper [6] an approach for online stabilization of anthropomorphic robot walking on uneven surface is presented. Robust control design for balance based on Zero Moment Point (ZMP), reference map, robust trajectories of center of mass are proposed. The result of method simulation is stability of walking and decreasing of trajectories errors.

In the paper [7] walking trajectory generation method, which includes free leg trajectory and ZMP methods, is considered. The method was tested on humanoid robot HRP-2. The test results show that balance was improved due to changing of ZMP at the expense of changing the foot position.

The purpose of this paper is to analyze methods for ensuring the sustainable position and movement of humanoid robots in space. On the basis of the analysis, the most suitable method will be proposed for the subsequent use in the Russian anthropomorphic robot Antares (Fig. 1).

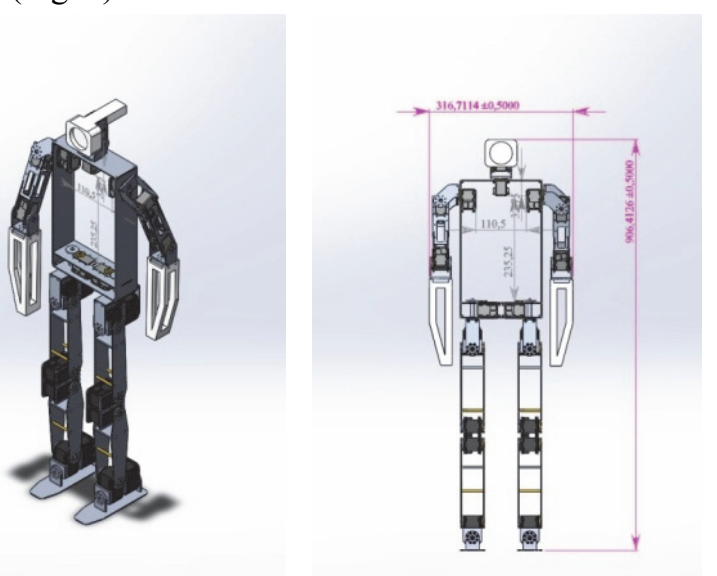

Fig. 1. The kinematic model of robot Antares.

\footnotetext{
* Corresponding author: denisov@iias.spb.su
} 
The robot Antares is unique in that it has two-motor knees, which provides larger angles of rotation to the engines, flexibility and plasticity of the leg assembly in comparison with analogues [8]. The two-motor knee greatly complicates the kinematic scheme, so it is necessary to carefully select the solutions ensuring the stability of the position of this robot [9].

\section{Analysis of methods to ensure the stability of the walking robot}

The result of contemporary balance control methods analysis for legged robots is five more perspective methods, which are considered in this section. This methods formalization, solved problems and necessary computing power are analyzed further.

\subsection{The parallel algorithm of the network operator method}

In the work [10] for solving the problem of stabilization of a bipedal walking robot on the basis of its inertial properties, a two-link inverse pendulum model was used to represent the robot. The idea of controlling such a pendulum lies in the redistribution of the angular momentum of the translational motion into rotational motion and vice versa. This approach allows reducing the moment on the side of the support and to control the sign and magnitude of the moment of gravity. The authors of the article regard a two-link inverse pendulum (shown in Fig. 2) as the control object, the hinge axes of which are parallel and allow the pendulum to move in the vertical plane.

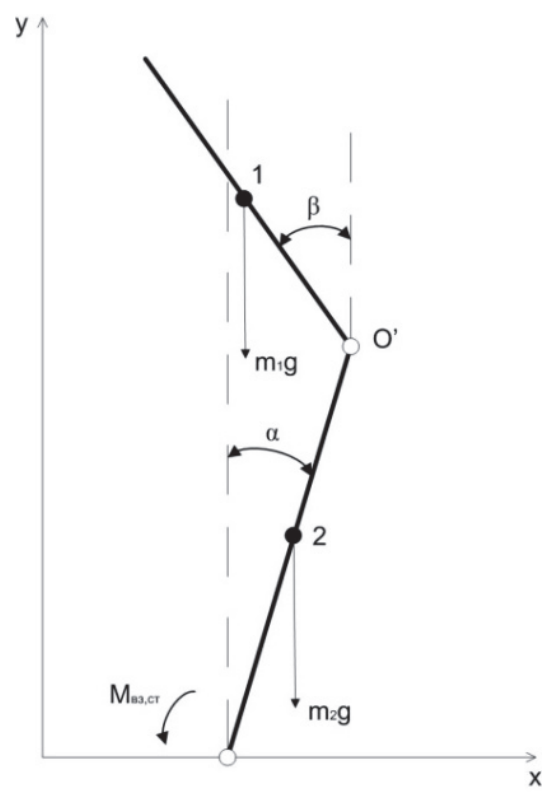

Fig. 2. Model of a two-link reverse pendulum.

In Figure $1, l 1, l 2$ is the length of the rods; $\mathrm{m} 1, \mathrm{~m} 2$ are the masses of rods 1 and 2, respectively. The mass of each rod is distributed evenly along the entire length. The control action is the angle of rotation of the rod 1 with respect to the vertical $\beta$.
The authors of the article proposed a formula for the mathematical model of the research object:

$$
\begin{aligned}
& \ddot{\alpha}=\frac{1}{K_{\alpha}(\alpha, \beta)}\left[-K_{\beta}(\alpha, \beta) \ddot{\beta}+K_{c m \alpha} \alpha+K_{g \alpha} \sin (\alpha)+\right. \\
& \left.+K_{g \beta} \sin (\beta)+M_{s}+m_{1} \frac{l_{1} l_{2}}{2}\left(\dot{\alpha}^{2}-\dot{\beta}^{2}\right) \sin (\alpha-\beta)\right]
\end{aligned}
$$

where $M_{s}$ is the moment from the side of the support surface; $K$ is the angular momentum; A, $\beta$ - angles of rotation; $K_{\text {ста }}$ is a constant coefficient of stabilization with respect to the angle $\alpha$.

The authors of the work assume that the kinematic control of the reverse pendulum is carried out by setting the desired angle $\left(\beta_{\mathrm{d}}\right)$. $\beta_{\mathrm{d}}$ differs from the real angle $\left(\beta_{\mathrm{r}}\right)$. The dynamics of the actuator drawing the angle $\beta$ is described by the following formula:

$$
\ddot{\beta}_{r}=-\frac{2 \varepsilon}{T} \dot{\beta}_{r}-\frac{1}{T^{2}} \beta_{r}+\frac{K}{T^{2}} \beta_{d}
$$

The authors of the paper constructed a pendulum model in the state space and carried out a control search in the form of $u=g(x)$, which satisfies the constraints $\mathrm{u}^{-}$ $\leq \mathrm{u} \leq \mathrm{u}^{+}$and ensures a minimum of functionals.

$$
\begin{gathered}
J_{1}=\sqrt{\sum_{1}^{4} \mathrm{x}_{i}^{2}\left(t_{f}\right)+\sum_{i=1}^{4} \int_{0}^{t} \mathrm{x}_{i}^{2}\left(t_{f}\right) \rightarrow \min ,} \\
J_{2}=t_{f} \rightarrow \min , \\
t_{f}-\text { control time } t_{f}=\left\{\begin{array}{c}
t, \text { if }\|\mathrm{x}(t)\| \leq \varepsilon \\
t^{+}, \text {or else }
\end{array},\right.
\end{gathered}
$$

where $\varepsilon$ is the small positive value, $\mathrm{t}^{+}$is the maximum time of the control process.

To construct a network operator, the authors introduce four finite ordered sets of: the variables $\mathrm{X}=\left(x_{1}, \ldots, x_{\mathrm{p}}\right)$, the parameters $Q=\left(q_{1}, \ldots, q_{\mathrm{R}}\right)$, the unary operations $O_{1}=\left(p_{1}(z), \ldots, p_{\mathrm{w}}(z)\right)$ and the binary operations $O_{2}=\left(x_{0}\left(z^{\prime}, z^{\prime \prime}\right), x\left(z^{\prime}, z^{\prime \prime}\right), \ldots, x_{\mathrm{v}-1}\left(z^{\prime}, z^{\prime \prime}\right)\right)$.

A network operator is an oriented graph that describes mathematical expressions. The elements from sets of parameters $Q$ or variables $X$ correspond to any node. A binary operation from the set $\mathrm{O}_{2}$ corresponds to any node not being a source. An unary operation from the set $O_{1}$ corresponds to any curve of the graph.

In this paper, a network operator matrix was used. It was constructed using the adjacency matrix of the network operator graph to represent the network operator in the computer's memory. In the network operator matrix, off-diagonal non-zero elements indicate the numbers of unary operations, and the diagonal elements indicate the numbers of binary operations [11]. To calculate the result of a mathematical expression from the matrix of the network operator, the vectors of the node numbers of the input variables were introduced. The search for the network operator matrix was made using a genetic algorithm.

A numerical experiment was performed, as a result of which a parameter vector and a network operator matrix were obtained. The matrix corresponds to the following control law:

$$
y=-x_{1} q_{1}-x_{2} q_{2}+x_{3} q_{3}+x_{4} q_{4}
$$


As a result of using a parallel algorithm for synthesizing the stabilization system of a biped walking robot by the network operator method, a nonlinear control law was obtained with respect to the state coordinates, which satisfies the given quality.

\subsection{The spherical inverted pendulum mode}

In the paper [12], the spherical inverted pendulum model for the biped robot balance control is considered. It describes the system using the two rotational degrees of freedom at the ankle.

The authors described an energy-based control architecture for push recovery on humanoid robots. The idea of this method consists in transforming the SIP model into a critically damped system with the use of a single tuning parameter.

A stable walking gate was generated and controlled by the SIP model. The authors used the principles of passive dynamic walking. The SIP model was described as a hybrid dynamic system. Two stages of this dynamic system are continuous dynamic model for the single support phase and during the exchange of support instance and impact of the swing leg. To restore any lost energy an internal impulse was used.

The result of robot walking simulation is the vertical motion with the same periodicity as that of the human walk. This approach is different in that the motion of the CoM is not constrained to a constant height of the plane during a single-step motion resulting in a more natural overall motion for the entire humanoid body.

\subsection{The method of natural synergies}

The paper [13] considers the possibility of controlling the movement of a two-link anthropomorphic robot on the basis of coordination of natural synergies. The authors explore an approach demonstrating the advantages of motor control, which is based on the management of their own movements, which is a natural synergy. Motions occur along the eigenvectors of the dynamic equation of the mechanical system. These are combinations of joint angles and moments, which is an advantage, since it greatly simplifies the development.

To analyze the robot's dynamics, its model is considered in the form of a two-link reverse pendulum, where the upper link represents the body, head and hands, and the lower link - legs [9, 14]. In numerical calculations, the following parameters of the anthropomorphic robot were used: weight $-70 \mathrm{~kg}$, height $-170 \mathrm{~cm}$.

The authors consider the control of the robot motion in terms of natural synergies. With the help of the linear approximation, it is possible to decompose the motion into components directed along two eigenvectors, which allows us to derive two scalar equations describing the motion of a single-element inverse pendulum controlled by its PD controller.

$$
\lambda_{i} \ddot{\xi}_{i}(t)=\eta_{i}^{\text {con }}(t), i=A, H
$$

$$
\begin{aligned}
& \eta_{i}^{\text {con }}(t)=\eta_{i}^{\text {con }}(t-\tau)+S_{i}^{e i g}\left(\xi_{i}^{\text {des }}(t)-\xi_{i}(t-\tau)\right)- \\
& -V_{i}^{e i g} \dot{\xi}_{i}(t-\tau)-\lambda_{i} \ddot{\xi}_{i}(t-\tau)
\end{aligned}
$$

In the case of a single-faced inverted pendulum $\lambda=J / m g h, \eta=T / m g h$,

$S_{\mathrm{i}}^{\text {eig }}=S / m g h, V_{\mathrm{i}}^{\text {eig }}=V / m g h$, where $J$ is the moment of inertia of the pendulum relative to the axis of rotation; $m$ is its mass; $h$ is the height of the position of the center of mass above the axis of rotation; $g$ is the acceleration of gravity; $T$ is the total moment of forces (except gravitational) relative to the axis of rotation; $S$ and $V$ are parameters of the PD controller.

In the work, stiffness and viscosity matrices were obtained in terms of joint angles. The resulting matrices are symmetric, but not diagonal. Thus, the authors conclude that the management of articular angles depends not only on the current values of a given angle, but also on the angles in all other joints, which determines the implementation of the PD controller, in which correction for each degree of freedom is determined by the values of the coordinates and their derivatives for all degrees of freedom.

The authors of this article carried out an analysis of the stability of control and an experiment on stability of the robot PostuRob II, the parameters of which are close to human. PostuRob II consists of a body and two legs. Connection between the elements of the robot is carried out by means of the hip and ankle joints [15]. During the experiment, signals from mechatronic sensors of joint angles came to the computer in real time.

The independence of the PD controller of each of the natural synergies allowed us to analyze the stability of the entire model through a separate analysis of the stability of the movement of each of these synergies.

The parameters of the PD controller have been calculated and tested at work. The selected parameters provide stability of movements for both the robot and the model. Thus, the movements of the lower segment, as required, compensated for the displacement of the center of gravity associated with the movements of the upper segment.

The study confirmed sustainable management on a real robot by the principle of independent control of each of the natural synergies with feedback loop parameters during a real experiment.

\subsection{A dual length linear inverted pendulum method}

In the paper [16] the method for obtaining walking parameters for biped robot moving along a slope is considered. The authors investigate a strategy to realize biped robot walking along a slope, when robot's centre of mass (CoM) is moved up or down during movement. This is because the height of the pendulum is kept at the same length on the left and right legs. Thus, one of this method purposes is to bring the CoM up to higher ground. In this method a dual length linear inverted pendulum method (DLLIPM) is used. DLLIPM is when 
a different height of pendulum is applied on the left and right legs of a robot.

As a result of using the method of walking along a slope based on DLLIPM and Newton Raphson algorithm, symmetrical motions and smooth walking trajectories were achieved and the maximum force fluctuation was reduced. In the future, the authors will expand the concept of DLLIPM for diagonal walking, turning on a slope and stairs.

\subsection{The method of fuzzy control}

The method of overcoming the "distance" between the theory of adaptive and robust control with the practice of its application, based on the transition to multialternative systems, is considered in [17].

The ideas of multi-alternativeness have biological analogies, defined in W. Ashby's Law of Requisite Variety [18], and also correspond to fuzzy control procedures of L. Zadeh [19].

The authors regard the inverted pendulum model as a typical member of structurally unstable objects. The model is fixed on the platform moving in a horizontal plane with some friction under the action of the external force (Fig. 3).

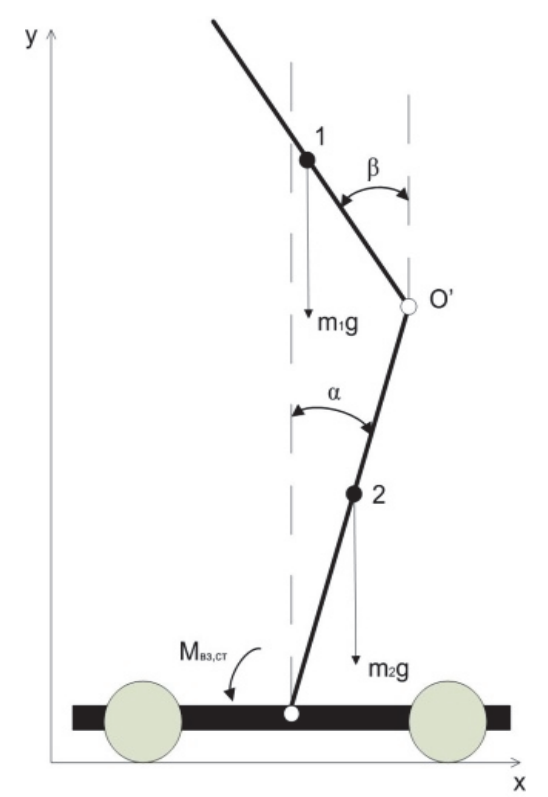

Fig. 3. Model of a moving platform with pendulum.

The movement of an unstable object is given as:

$\left\{\begin{array}{c}\left(m_{1}+m_{2}\right) \ddot{\mathrm{x}}-m_{2} L\left(\ddot{\alpha} \cos \alpha-\dot{\alpha}^{2} \sin \alpha\right)=F-F_{f r} \\ F_{f r}=\mu\left[m_{1} g+m_{2} \cos \alpha\left(g \cdot \cos \alpha-\ddot{\mathrm{x}} \sin \alpha-\dot{\alpha}^{2} L\right) \operatorname{sign}(\dot{\mathrm{x}})\right] \\ -\ddot{\mathrm{x}} \cos \alpha+L \ddot{\alpha}-g \sin \alpha=0\end{array}\right.$

where $m_{1}, m_{2}$ are the masses of platform and inverted pendulum, $\mathrm{kg}$; $L$ is the length of pendulum, $\mathrm{m} ; x$ is the coordinate of platform's horizontal location, $\alpha$ is the lack of perpendicularity angle, rad; $g$ is the acceleration of gravity, $\mathrm{m} / \mathrm{s} 2 ; \omega$ is the angular rate, $\mathrm{rad} / \mathrm{sec} ; \mu$ is a dry friction coefficient of the platform; $F_{\mathrm{fr}}$ is the dry friction force, $\mathrm{N} ; F$ is the imposed force to the platform, $\mathrm{N}$.
For stabilization of the inverted pendulum the authors used the structure of the system with a regulator, on which the data about object condition is received at the input, and at the output - a signal generated into the control action on the object.

For the synthesis of the fuzzy regulator structure the authors of this article crate the catalogue (list) of linguistic and fuzzy input and output variables of the regulator; form the functions of these variables attachment; create solving rules of the fuzzy logic output determining the object control.

Multicriteriality is the main feature of creating the control algorithm. It is the control of two object coordinates, pendulum angular deviation $\alpha$ and the position of the platform $x$ by one actuation. Thus, the idea of the movement control separation is used: when the angle and speed reach small values $z_{1}{ }^{2}$ and $z_{2}{ }^{2}$, platform condition control is being realized. In this case the angle and speed control becomes a priority, if $\alpha$ and $\alpha^{\prime}$ go beyond the small range. As a result of applying this method, ten fuzzy solving rules were obtained. These rules form evident signs and modules for control values, which lead pendulum coordinates to desired condition, realize forced pendulum angular deviation to desired platform movement, lead platform to desired location $x_{0}$, form result control actuation which corresponds to the present object state.

As a result of the solved rules the authors received a fuzzy set:

$$
\mu_{u}^{*}(y)=\sum_{j=1}^{10} \mu_{u}^{*}(y)
$$

Balance and robust properties of the synthesized system were tested on the inverted pendulum model, described earlier, with the parameters: $m_{1}=100 \mathrm{~kg}$, $m_{2}=20 \ldots 200 \mathrm{~kg}, L=5 \mathrm{~m}, g=9,81 \mathrm{~m} / \mathrm{sec} 2, \mu=0,05$, where mass $m_{2}$ changed from 20 to $200 \mathrm{~kg}$. Initial pendulum angular position is $\alpha(0)=0.2 \mathrm{rad}$, platform position $-\mathrm{x}(0)=0$.

Stabilization is realized by corresponding platform movements. The time of the platform returning to initial position after ending of angular stabilization is $35 \mathrm{sec}$ in both cases.

The received results confirm efficiency of fuzzy control by using multialternative systems in object control problems in critical modes.

\section{Discussion}

As a result, five different methods of balance control for legged robots were considered in the research. Also during the survey the main parameters, which should be taken during kinematic modelling was discovered (Table 1). Most of them are generally used for solving balance problem of legged robot.

We reviewed the parallel algorithm of the network operator method, the method of natural synergies, the method of fuzzy control, the spherical inverted pendulum mode and a dual length linear inverted pendulum method (DLLIPM). 
Table 1. General parameters.

\begin{tabular}{|c|c|c|}
\hline $\begin{array}{c}\text { Physical } \\
\text { parameter }\end{array}$ & Symbol & Unit \\
\hline Body mass & $\mathrm{m}$ & $\mathrm{kg}$ \\
\hline Body length & 1 & $\mathrm{~m}$ \\
\hline Body inertia & $\mathrm{J}$ & $\mathrm{Kg}^{*} \mathrm{~m}^{2}$ \\
\hline Gravity & $\mathrm{g}$ & $\mathrm{m} / \mathrm{s}^{2}$ \\
\hline Friction & $\mathrm{F}_{\mathrm{fr}}$ & $\mathrm{N}$ \\
\hline Angle & $\beta$ & $\mathrm{rad}$ \\
\hline Turning angle & $\beta_{\mathrm{r}}$ & $\mathrm{rad}$ \\
\hline Angular velocity & $\omega$ & $\mathrm{rad} / \mathrm{sec}$ \\
\hline Coordinates & $\mathrm{x}, \mathrm{y}$ & $\mathrm{m}$ \\
\hline
\end{tabular}

The robot model was presented as an inverted pendulum, which corresponds to an unstable position in the space. All the methods were tested on the robot model and proved to be efficient. Conclusions and summary about analyzed methods are presented in Table 2 .

Table 2. The analyzed methods of balance control.

\begin{tabular}{|c|c|c|c|c|}
\hline № & Method & $\begin{array}{c}\text { Solving } \\
\text { problem }\end{array}$ & $\begin{array}{c}\text { Algorithm and } \\
\text { solving } \\
\text { complication } \\
\text { (resources } \\
\text { consumption) }\end{array}$ & $\begin{array}{c}\text { Time of } \\
\text { inverted } \\
\text { pendulum } \\
\text { balancing }\end{array}$ \\
\hline 1 & $\begin{array}{l}\text { the } \\
\text { parallel } \\
\text { algorithm } \\
\text { of the } \\
\text { network } \\
\text { operator } \\
\text { method }\end{array}$ & $\begin{array}{c}\text { Balance } \\
\text { control of } \\
\text { humanoid } \\
\text { robot } \\
\text { walking }\end{array}$ & normal & $1.6 \mathrm{sec}$ \\
\hline 2 & $\begin{array}{c}\text { the } \\
\text { spherical } \\
\text { inverted } \\
\text { pendulum } \\
\text { mode }\end{array}$ & $\begin{array}{c}\text { Balance } \\
\text { control of } \\
\text { humanoid } \\
\text { robot } \\
\text { walking }\end{array}$ & normal & $2.1 \mathrm{sec}$ \\
\hline 3 & $\begin{array}{c}\text { the } \\
\text { method of } \\
\text { natural } \\
\text { synergies }\end{array}$ & $\begin{array}{l}\text { Balance } \\
\text { and } \\
\text { motion } \\
\text { control of } \\
\text { humanoid } \\
\text { robot } \\
\text { standing }\end{array}$ & high & $1.2 \mathrm{sec}$ \\
\hline 4 & $\begin{array}{c}\text { the } \\
\text { method of } \\
\text { fuzzy } \\
\text { control }\end{array}$ & $\begin{array}{l}\text { Balance } \\
\text { control of } \\
\text { humanoid } \\
\text { robot } \\
\text { standing }\end{array}$ & high & $1.7 \mathrm{sec}$ \\
\hline 5 & DLLIPM & $\begin{array}{l}\text { Balance } \\
\text { control of } \\
\text { humanoid } \\
\text { robot } \\
\text { walking } \\
\text { along } \\
\text { slope }\end{array}$ & normal & $1.8 \mathrm{sec}$ \\
\hline
\end{tabular}

The method presented in [13] was tested on the real robot and showed the best balance control of a humanoid robot. However, this method is very resource-intensive one. It requires a lot of computational capability, so it cannot be used in embedded systems such as the anthropomorphic robot Antares. Given that, the method presented in [10] will be used in the development of Antares. This method showed the second result in balance control tests. The advantages of the method include operation speed; the ability to control the moment value of gravity force and to decrease the support moment.

\section{Conclusion}

The balance problem for walking legged robots is considered. The perspective methods for kinematic control are selected. The main parameters influencing for stable motion of legged robot are discovered. The future work will be related to the integration of the programming solutions of balance control method to the robotic core of the robot Antares [20-22].

The presented work was supported by the Russian Science Foundation (grant No. 16-19-00044).

\section{References}

1. E. Najafi, G.A.D. Lopes, R. Babuska, 19th World Congress IFAC, 2177-2182 (2014)

2. M. Shahbazi, G.A.D. Lopes, R. Babuska, 19th World Congress IFAC, 2171-2176 (2014)

3. D. Sarkara, S.K. Dubeya, A. Mahapatrab, S.S. Roy, 2nd International Conference ICIAME 2014, 93-99 (2014)

4. Z. Shia, Q. Zhang, J. Tu, Z. Wang, X. Zhang, International Workshop IWIEE, 3250-3255 (2012)

5. A. Escande, A. Kheddara, S. Miossec, Robotics and Autonomous Systems. 61, 428-442 (2013)

6. N. Wu, C. Chew, A. Poo, R. Li, 6th IFAC Sym. on Mech. Sys. Hangzhou, China, 577-582 (2013)

7. K. Nishiwaki, S. Kagami, IFAC Proceedings Volumes. 39, 675-700 (2006)

8. A.S. Kodyakov, N.A. Pavlyuk, V.Yu. Budkov, R.A. Prakapovich, IOP Conf. Series: Journal of Physics: Conf. Series. 803, 012074 (2017)

9. N. Pavluk, A. Ivin, V. Budkov, A. Kodyakov, A. Ronzhin, First International Conference ICR 2016, 113-123 (2016)

10. B.B. Kulakov, A.S. Zharkova, D.E. Kazaryan, RUDN Journal of engineering researches. 4, 26-36 (2010)

11. I. Vatamaniuk, G. Panina, A. Saveliev, A. Ronzhin, IEEE Int. Conf. on Autonomous Robot Systems and Competitions, 306-310 (2016)

12. A. Elhasairi, A. Pechev, Front. Robot AI, 2(21) (2015)

13. A.V. Aleksandrov, T. Mergner, A.A. Frolov, G. Hettich, I.P. Muravev, Russian Journal of Biomechanics. 17, 1(59), 94-109 (2013) 
14. A. Ronzhin, I. Vatamaniuk, N. Pavliuk, 2016 Int. Conf. and exposition on electrical and power engineering, 926 (2016)

15. L. Assländer, G. Hettich, T. Mergner, Human Movement Science. 41, 147-164 (2015)

16. F. Ali, A.Z. Hj. Shukor, M.F. Miskon, M.K.M. Nor, S.I.Md Salim, International Journal of Advanced Robotic Sysems. 10(377) (2013)

17. E.M. Vasiljev, D.M. Prokofjeva, Fuzzy control of structurally unstable objects. VSTU Journal. 8, №10 (1), 8-12 (2012)

18. W.R. Ashby, Publishing of foreign literature. 432 (1959)

19. L.A. Zadeh, Outline of a new approach to the analysis of complex systems and decision processes. (M.: Znanie. 64 1974)

20. L.Yu. Vorochaeva, A.S. Yatsun, S.F. Jatsun, SPIIRAS Proceedings. 3(52) (2017)

21. A. Ronzhin, A. Saveliev, O. Basov, S. Solyonyj, Springer-Verlag Berlin Heidelberg, A. Ronzhin et al. (Eds.): ICR 2016, LNCS/LNAI, 9812 (2016)

22. A. Ivin, D. Mikhalchenko. 21st Fruct Conf. (2017) 